Cahiers $d u$ MONDE RUSSE

\section{Cahiers du monde russe}

Russie - Empire russe - Union soviétique et États indépendants

$53 / 4 \mid 2012$

Varia

\title{
Valérie Pozner, Natacha Laurent, éds., Kinojudaica
}

\section{Thomas Chopard}

\section{OpenEdition \\ Journals}

Édition électronique

URL : http://journals.openedition.org/monderusse/7752

DOI : $10.4000 /$ monderusse. 7752

ISSN : $1777-5388$

Éditeur

Éditions de l'EHESS

Édition imprimée

Date de publication : 15 décembre 2012

ISSN : $1252-6576$

\section{Référence électronique}

Thomas Chopard, «Valérie Pozner, Natacha Laurent, éds., Kinojudaica », Cahiers du monde russe [En ligne], 53/4 | 2012, mis en ligne le 02 décembre 2013, Consulté le 25 septembre 2020. URL : http:// journals.openedition.org/monderusse/7752 ; DOI : https://doi.org/10.4000/monderusse.7752

Ce document a été généré automatiquement le 25 septembre 2020.

(c) École des hautes études en sciences sociales 


\title{
Valérie Pozner, Natacha Laurent, éds., Kinojudaica
}

\author{
Thomas Chopard
}

\section{RÉFÉRENCE}

Valérie POZNER, Natacha LAURENT, éds., Kinojudaica. Les représentations des Juifs dans le cinéma de Russie et d'Union soviétique des années 1910 aux années 1980. Paris : Nouveau monde éditions, 2012, 585 p.

$1 \quad$ Il est des ouvrages dont le sujet en apparence réduit semble ne s'adresser qu'à quelques spécialistes, et qui recèlent en réalité de multiples facettes. Au confluent de l'histoire du cinéma, de l'histoire sociale et de l'histoire des nationalités, Kinojudaica est de ceuxci. Les treize contributions de chercheurs russes, ukrainiens, anglo-saxons et français, rassemblées par Valérie Pozner et Natacha Laurent se concentrent, à une exception près, sur les années comprises entre 1910 et 1960. Les auteurs font jouer de multiples façons les deux objets «juifs » et "cinéma " - les juifs travaillant dans le cinéma, les juifs comme public de cinéma et surtout les juifs comme sujets de films de fiction ou documentaires - pour opérer des études de cas qui s'intègrent chacune dans des problématiques plus générales.

2 L'ouvrage s'ouvre sur la question de savoir s'il a existé dans l'Empire russe puis en Union soviétique, un cinéma proprement juif. Les réponses apportées varient entre les études générales et la retranscription du parcours de réalisateurs juifs. La création d'un cinéma juif répond avant tout à la demande d'un public yiddishophone. V. Pozner propose dans le premier chapitre une analyse d'ensemble des différentes productions juives dans la zone de résidence de l'Empire russe et l'émergence au début du $\mathrm{xx}^{\mathrm{e}}$ siècle d'une thématique juive dans le cinéma russe. Toute l'ambivalence du « juif » au cinéma est posée d'emblée : le sujet s'adresse à la population juive, mais il est aussi investi par le cinéma russe. Les juifs au cinéma ne sont jamais présentés comme une niche: de même que la production juive entre souvent en concurrence avec les productions issues 
des autres nationalités de la région, le thème en lui-même dépasse aussi les limites de la seule nationalité judéo-soviétique pour s'ouvrir aux autres nationalités, voire à l'international. Ce dialogue est notamment étudié par Claire Le Foll dans le cas du cinéma biélorusse. Dans le cadre particulier de la politique des nationalités en Biélorussie, qui reconnaît comme langues officielles, outre le biélorusse, le yiddish, le russe et le polonais, l'émergence d'un cinéma yiddish à partir de 1925 entre en contradiction avec la politique de biélorussisation opérée par les autorités dans le même temps. Le cinéma juif sert donc régulièrement dans l'ouvrage de révélateur des à-coups et des contradictions de la politique soviétique des nationalités. À travers le cinéma, la culture yiddish trouve une forme d'institutionnalisation que le pouvoir soviétique lui conteste régulièrement.

3 Cette difficile intégration de la culture juive dans la culture soviétique est aussi analysée dans les portraits de deux réalisateurs juifs : Mihail Romm et Mihail Dubson. Ces deux chapitres abordent sous un angle différent la difficulté de filmer des juifs. Le premier réalisateur, Romm, n'aborde ce thème que de façon incidente dans ses films ; son engagement n'est pas artistique mais au final politique, dans une lutte contre l'antisémitisme. Le second portrait, celui de Dubson, illustre les difficultés opposées par les institutions dans les années 1930 à un réalisateur qui tente de travailler frontalement le thème juif dans ses films. On ne peut que souligner le travail minutieux dans les archives qui permet de retranscrire précisément les négociations, les critiques et les conditions opposées par la Direction centrale du cinéma et de la photographie. Outre la question du thème juif au cinéma, l'histoire des productions présentée par Kinojudaica relève aussi d'une histoire sociale des productions artistiques. La présence des juifs à l'écran dépend d'une négociation permanente qui conditionne et infléchit les œuvres d'art elles-mêmes. Cette étude de la genèse des œuvres ouvre, dans l'ouvrage, sur une attention constante aux détails filmiques : allusions, élisions, métaphores sont soulignées comme autant de détours pris par les cinéastes.

4 Ces détours tiennent aussi à la juxtaposition de multiples discours ; l'investissement des réalisateurs entre en concurrence, parfois en conflit, avec les exigences idéologiques posées par les institutions soviétiques et souvent même intégrées par les cinéastes euxmêmes. L'une des thématiques continues de l'ouvrage est celle de la « fabrique » du juif soviétique. Que ce soit dans les films de fiction produits en Biélorussie ou, plus clairement encore, dans les documentaires de propagande sur le Birobidžan (étudié par Aleksandr Ivanov) et les paysans juifs nouvellement installés sur la terre (Eric Aunoble), la seule figure acceptable du juif soviétique semble être celle qui abandonne toute trace de particularisme juif et yiddish au profit de l'internationalisme. L'analyse des œuvres évite tout discours abstrait sur le cinéma de propagande soviétique, et montre au contraire la diffusion variable et parfois même les échecs des discours de propagande. Ainsi, Aunoble souligne les variations du propos et l'esthétisation du discours, selon que l'œuvre s'adresse aux seuls Soviétiques ou au contraire à l'international.

Allers et retours entre films documentaires et films de fiction permettent aussi de saisir la place centrale occupée par le cinéma dans la propagande soviétique. Le premier chapitre, dû à V. Pozner, est consacré directement à la propagande et à la lutte contre l'antisémitisme. L'étude de la constellation d'archives relatives à cette lutte contre l'antisémitisme - brochures, journaux, etc. - offre un tableau minutieux des efforts du pouvoir soviétique pour combattre les regains d'antisémitisme en URSS, au temps de la guerre civile et dans les années 1920. Le cinéma sert ici de point d'ancrage à une étude 
plus vaste. L'auteur renverse aussi la perspective et souligne l'importante variation que connaît, à partir des années 1930, la figure du juif mise en avant par cette propagande. Le juif était, en partie positivement, «non assimilable, rétrograde, individualiste, rêveur. Il devait désormais être montré comme flemmard, asocial, accapareur, finir comme assassin et contre-révolutionnaire » (p. 174).

6 Le corpus de films offre en effet un large panorama des représentations du cadre de vie des juifs. À travers les études de cas, le lecteur plonge à plusieurs reprises dans les shtetls russes puis soviétiques, soumis au déclin (Le bonheur juif, Le retour de Nathan Becker), dans l'Odessa fantasmée en « mère du crime » d'Isaac Babel (Benia Krik) ou dans une ville indéterminée où se met en place l'extermination des juifs locaux (Les Insoumis).

7 La Shoah et ses représentations à l'écran constituent un segment à part de l'ouvrage. Étant donné la quasi-interdiction de représenter l'extermination des juifs soviétiques à l'écran, les films sont rares, et le travail de construction du corpus est ici précieux : sont juxtaposés l'étude des images documentaires présentées au Tribunal de Nuremberg, le seul film de fiction des années 1940 traitant directement du sujet (Les Insoumis), et un documentaire de commande sur le procès de neuf membres du Sonderkommando 10-a tenu à Krasnodar en 1963 ( $\mathrm{Au}$ nom des vivants). Ce dernier cas, étudié par Vanessa Voisin, est l'occasion de revenir longuement sur ce silence à l'égard du génocide, instauré par la répression des rares élites yiddish après-guerre; malgré des allusions évidentes, le film n'emploie d'ailleurs jamais le mot «juif», ce qui ne l'empêche pas d'évoquer pleinement l'expérience de guerre du Sonderkommando et des populations civiles. De la même façon, Les Insoumis de Mark Donskoj crypte en large partie l'évocation du massacre de Babij Jar, en s'appuyant sur un roman à succès dont l'action se déroule dans le Donbass. Au nom des vivants est aussi l'occasion de souligner combien ce documentaire est motivé par un contexte international modifié : le dégel, le procès Eichmann et une dénonciation d'une RFA belliciste et consumériste. L'analyse de la mise en scène du procès montre aussi combien le film s'inscrit dans la longue histoire des films de procès soviétiques, malgré un propos profondément renouvelé. Les images présentées au procès de Nuremberg présentent enfin un contrepoint important aux nombreux travaux sur les films américains sur les camps de concentration; les films soviétiques offrent une configuration singulière, traitant ouvertement des camps d'extermination, mais laissant les témoins au second plan. L'essentiel du propos tenait, non pas dans la reconnaissance des victimes, mais dans un discours percutant de dénonciation de l'ennemi. Il faut en effet attendre Au nom des vivants pour que les témoins soient directement mis en scène dans leur confrontation aux bourreaux.

8 L'ouvrage est dense. Il présente tout d'abord au lecteur l'attrait de la curiosité relative aux films, souvent oubliés. Cet attachement aux images se retrouve dans les nombreuses illustrations tirées de films qui ponctuent les chapitres et renforcent le propos. Les études sont fines, claires, et ouvrent sur des réflexions centrales de l'historiographie : l'histoire sociale des productions et des administrations, l'histoire culturelle des représentations, l'histoire des politiques soviétiques de nationalités, l'histoire de l'antisémitisme, de la Shoah et des violences de guerre en général. On peut regretter l'absence de bibliographie générale en fin de volume qui aurait souligné l'ample historiographie mentionnée dans les notes; voire l'absence d'un DVD. Les auteurs ont préféré aux ouvrages une importante filmographie, de près de cent pages, des films documentaires et de fiction ayant un rapport direct avec les juifs. Cette 
longue liste témoigne du profond investissement dans le matériau filmique opéré par les différents chercheurs qui, jamais, ne discourent en surplomb et offrent toujours à voir l'intérêt des productions soviétiques. 